\title{
Dientes Supernumerarios Suplementarios en un Paciente Adulto Parcialmente Dentado: Reporte de Caso
}

\author{
Supplementary Supernumerary Teeth in a Partially Edentulous Adult Patient: Case Report
}

\author{
Ramón Fuentes ${ }^{1,2}$; Giannina Álvarez ${ }^{3}$; Ivonne Garay ${ }^{1}$; Alain Arias ${ }^{1,2,4}$ \& Fernando José Dias ${ }^{1,2}$
}

FUENTES, R.; ÁLVAREZ, G.; GARAY, I.; ARIAS, A. \& DIAS, F. J. Dientes supernumerarios suplementarios en un paciente adulto parcialmente dentado: Reporte de caso. Int. J. Morphol., 36(2):478-482, 2018.

RESUMEN: Aquellos casos en que se excede la cantidad normal de dientes para la fórmula primaria o definitiva se conocen como dientes supernumerarios o hiperodoncia. Esta puede presentarse de manera aislada o múltiple, unilateral o bilateral y afectar tanto a la maxila como a la mandíbula. Es más prevalente en hombres, en dentición permanente y en el maxilar, siendo el mesiodens el supernumerario encontrado con mayor frecuencia. Su etiología aún no está del todo clara, sin embargo, una hiperactividad de la lámina dental junto a una asociación de factores genéticos y ambientales sería la teoría más aceptada. Pueden ser asintomáticos y detectados solo en radiografías o pueden presentar manifestaciones clínicas como apiñamiento, retardo en la erupción, reabsorción radicular, diastemas entre otros. El tratamiento depende del tipo de diente y de su relación con las estructuras adyacentes. En el siguiente reporte presentamos el caso de un paciente de sexo masculino, de 54 años de edad, no sindrómico, desdentado parcial superior e inferior que acude a una consulta dental privada (Temuco) para solicitar tratamiento rehabilitador. En la radiografía panorámica de rutina se observan dos dientes supernumerarios, uno a cada lado de la mandíbula y en la región premolar. Al examinar este hallazgo con tomografía computarizada cone beam se determina que el supernumerario del lado derecho corresponde a un premolar suplementario y el de la izquierda a un supernumerario de morfología molariforme. En este caso se decide extraer los dientes debido a la localización de estos y a las implicancias con el tratamiento rehabilitador posterior.

PALABRAS CLAVE: Supernumerario; Hiperodoncia; Premolar; Diente suplementario.

\section{INTRODUCCIÓN}

La hiperodoncia o dientes supernumerarios corresponde a una anomalía del desarrollo dental de número, en donde se presentan uno o más dientes o estructuras odontogénicas adicionales a los 20 y 32 dientes de la serie normal de dentición primaria y permanente, respectivamente (Valmaseda-Castellón et al., 2001; Omer et al., 2010). La etiología de los dientes supernumerarios aún no está del todo clara y aunque en la literatura se presentan distintas teorías, la más aceptada se refiere a una actividad excesiva de la lámina dental durante etapas iniciales del desarrollo, en donde algunas vías de señalización molecular encargadas del control de estas etapas podrían alterarse y propiciar la formación de dientes supernumerarios (Ge \& Wang, 2013; Juuri $\&$ Balic, 2017).

También se ha asociado la presencia de dientes supernumerarios con algunos síndromes tales como la displasia cleidocranial, síndrome de Gardner y fisura labiopalatina
(Lubinsky \& Kantaputra, 2016); además de la interacción de factores genéticos, epigenéticos y ambientales (Montasser \& Taha, 2012; Juuri \& Balic). Pueden presentarse de forma única o múltiple, unilateral o bilateral y en cualquier lugar del arco dentario maxilar o mandibular, siendo más común en dentición permanente que primaria y más frecuentes en hombres que en mujeres (Juuri \& Balic). Los dientes supernumerarios se pueden clasificar de acuerdo a su posición en mesiodens, paramolares, parapremolares y cuartos molares, y de acuerdo a su forma en suplementarios o eumórficos y rudimentarios o dismórficos, pudiendo estos últimos ser de tipo cónico, tuberculado, molariforme y odontoma (Primosch, 1981; Garvey et al., 1999; Sebastián Sebastián et al., 2016). En el siguiente reporte de caso exponemos el hallazgo casual de dos dientes supernumerarios suplementarios en la mandíbula de un paciente adulto parcialmente desdentado y cuya ubicación hizo necesario considerar su manejo antes de comenzar un tratamiento rehabilitador con implantes.

\footnotetext{
${ }^{1}$ Departamento de Odontología Integral Adultos, Facultad de Odontología, Universidad de La Frontera, Temuco, Chile.

${ }^{2}$ Centro de Investigación en Ciencias Odontológicas (CICO), Facultad de Odontología, Universidad de La Frontera, Temuco, Chile.

${ }^{3}$ Programa de Magíster en Odontología, Facultad de Odontología, Universidad de La Frontera, Temuco, Chile.

${ }^{4}$ Universidad Adventista de Chile, Chillán, Chile.
} 


\section{REPORTE DE CASO}

Paciente masculino de 54 años, no sindrómico y que no reporta antecedentes médicos relevantes, acude a una consulta dental privada en la ciudad de Temuco, Chile, para realizar tratamiento rehabilitador con implantes. Al examen intraoral se observa desdentamiento parcial de la región posterior tanto en maxila como en mandíbula, sin otro ha- llazgo relevante. Se solicita de forma preliminar una radiografía panorámica de rutina en donde destaca el hallazgo de dos dientes de morfología similar a la de un premolar, ambos incluidos en el hueso de la región premolar derecha e izquierda (Fig. 1). Llama la atención la ausencia de cortical alveolar por sobre la región coronal del diente identificado

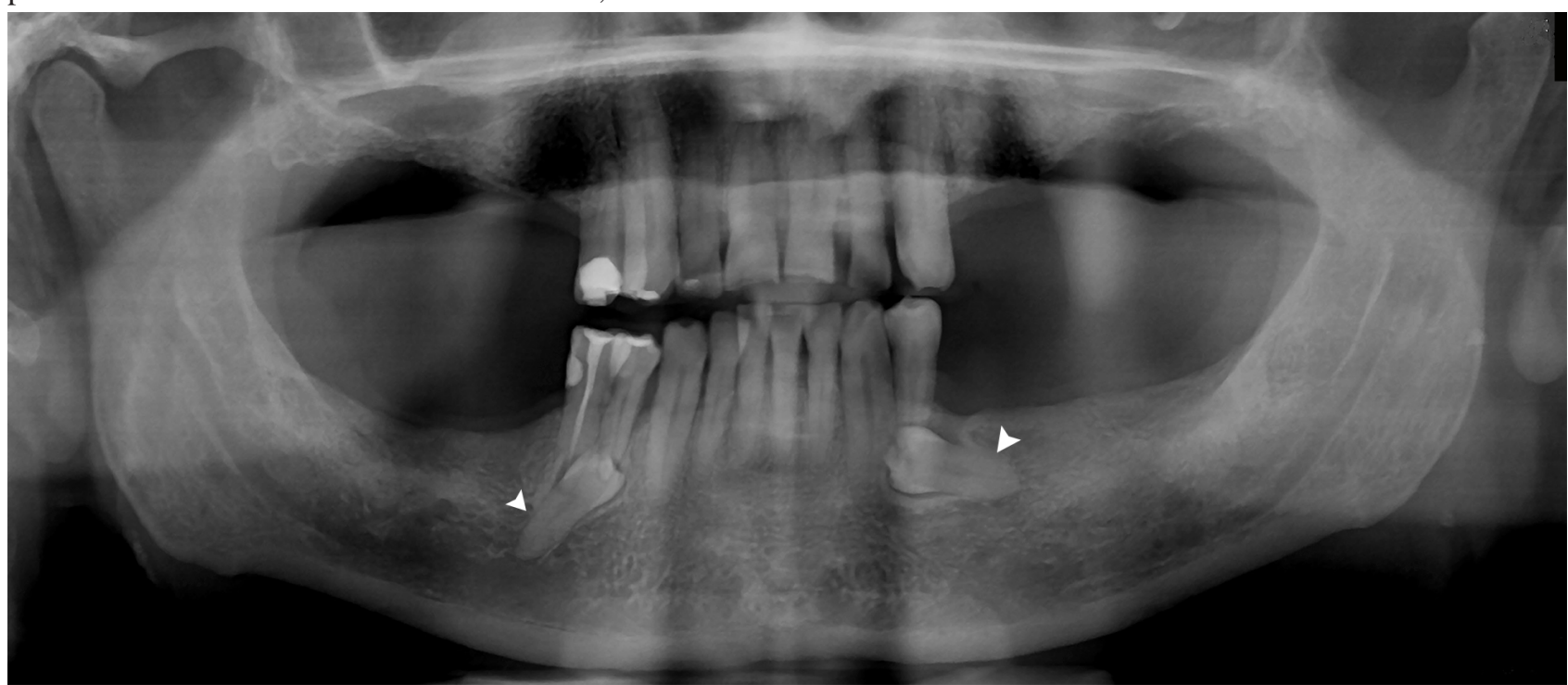

Fig. 1. Radiografía panorámica digital de paciente de sexo masculino, 54 años de edad, en la que se observa desdentamiento parcial de la región posterior de la maxila y mandíbula y presencia de dos dientes de morfología similar a la de un premolar, ambos incluidos en el hueso de la región premolar derecha e izquierda (flechas).

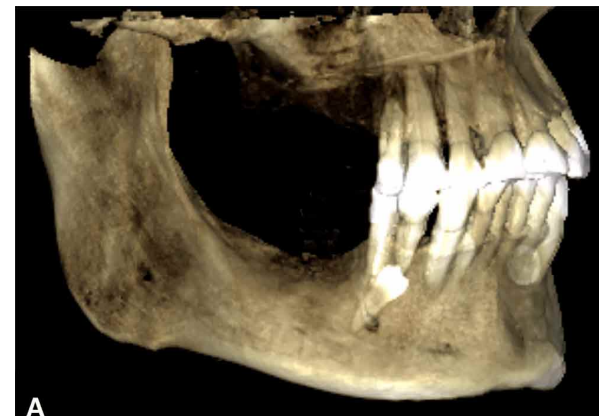

A

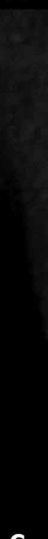

C
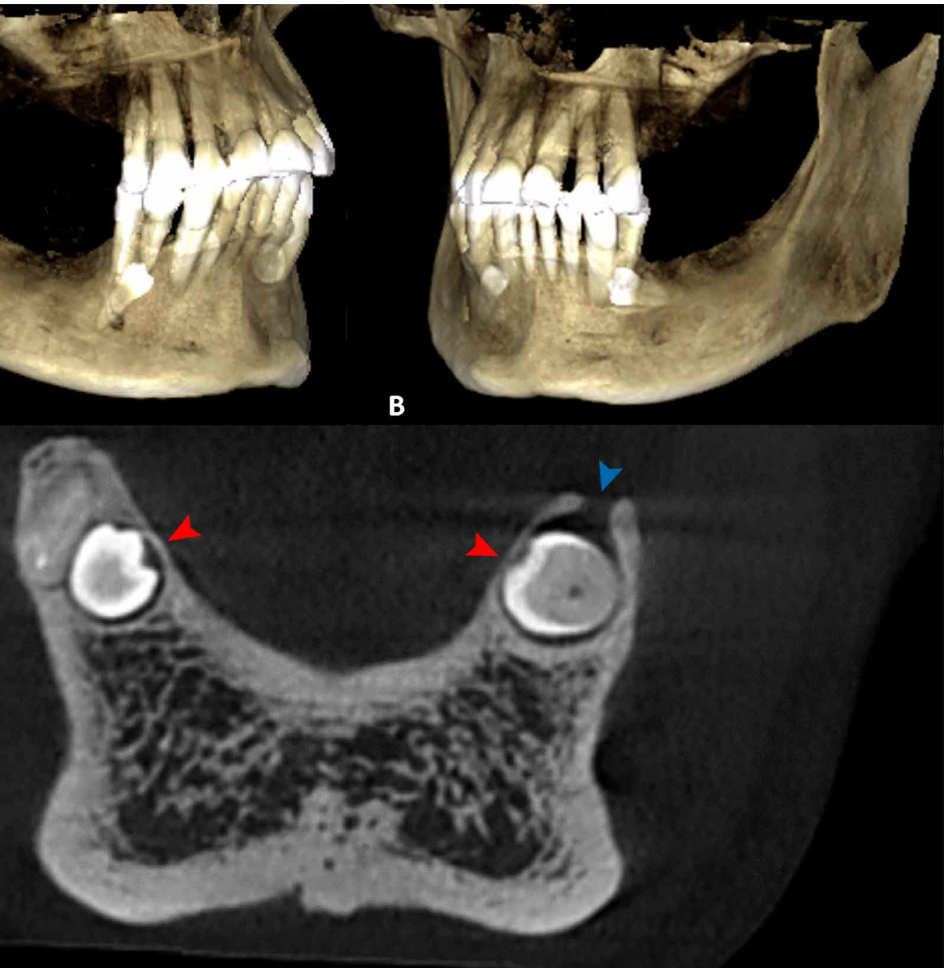

Fig. 2. A) Reconstrucción tridimensional (CBCT) en la que se observa la cara externa de la mandíbula del lado derecho y en donde se aprecia un diente supernumerario suplementario (tipo premolar) en posición mesioinclinada, caudal a los dientes 4.4 y 4.5 e impactado en el tercio apical de este último. B) Reconstrucción tridimensional (CBCT) en la que se observa la cara externa de la mandíbula del izquierdo y en donde se aprecia un diente supernumerario incluido, en posición horizontal, caudal a los dientes 3.4 y 3.3 y de morfología rudimentaria de tipo molariforme. C) Corte coronal en el CBCT a nivel de las coronas de los supernumerarios. Se observa una leve expansión y adelgazamiento de tabla ósea lingual en ambos lados (flecha roja) y una comunicación marginal de espacio pericoronario de supernumerario izquierdo (flecha azul). 
en el lado izquierdo y la ausencia del segundo premolar, lo cual provoca confusión para un diagnóstico final.

Con el objetivo de planificar el tratamiento rehabilitador con implantes y establecer implicancias o consideraciones que se deben tomar respecto del hallazgo con el tratamiento rehabilitador, se solicita una tomografía computarizada cone beam (CBCT) del área mandibular con un campo de visualización de 12 x $9 \mathrm{~cm}$ y tamaño de voxel 0,2 mm $(120 \mathrm{kV}, 10 \mathrm{~mA}, 24 \mathrm{~s}$ de adquisición). El examen fue realizado en el Servicio de Imagenología de la Clínica Odontológica Docente Asistencial (CODA) de la Universidad de La Frontera, con el tomógrafo PAX Zenith 3D (Vatech Co., Gyeonggi-Do, Korea). A través de este examen se establece que ambos dientes corresponden a dientes supernumerarios suplementarios. En la reconstrucción tridimensional del CBCT se observa que el diente identificado en el lado derecho se encuentra en posición mesioinclinada, caudal a los dientes 4.4 y 4.5 e impactado en el tercio apical de este último (Fig. 2.A). En el caso del diente del lado izquierdo este se observa incluido, en posición horizontal, caudal a los dientes 3.4 y 3.3 y de morfología rudimentaria de tipo molariforme (Fig. 2.B). En ambos lados se observa una leve expansión y adelgazamiento de tabla ósea lingual a nivel de las coronas de los supernumerarios, además de una comunicación marginal de espacio pericoronario en el supernumerario del lado derecho (Fig. 2.C). En una vista axial del CBCT se precisa que los dientes supernumerarios se encuentran en posición lingual respecto de sus vecinos (dientes 4.4 - 4.5 y 3.4 respectivamente) (Fig. 3.A).

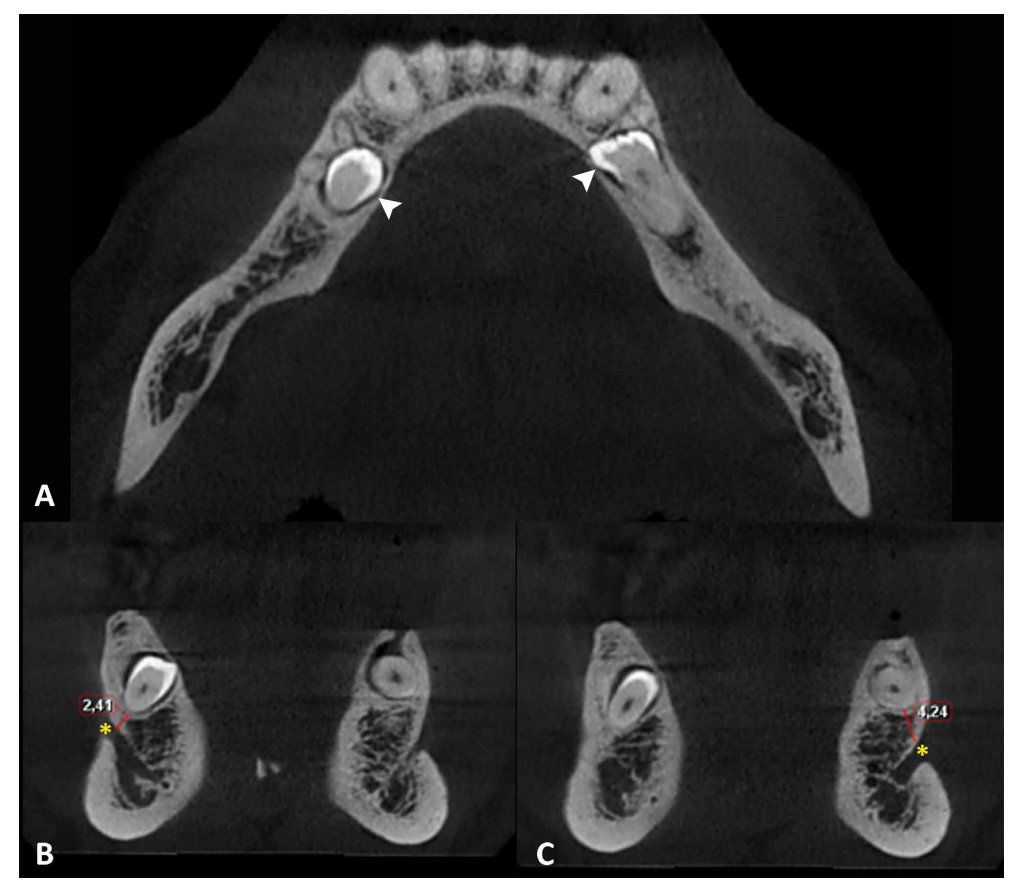

Fig. 3. A) Corte axial en el CBCT a nivel de tercio superior de cuerpo mandibular. Se observa la posición lingual de los dientes supernumerarios (flechas) respecto de los dientes 4.4 y 4.5 (asterisco) en el lado derecho y diente 3.4 (asterisco) en el izquierdo. B) Corte coronal en el CBCT a nivel del foramen mental (asterisco) en el lado derecho. C) Corte coronal en el CBCT a nivel del foramen mental (asterisco) en el lado izquierdo.
Por último, la distancia más corta entre el diente supernumerario del lado derecho y el foramen mental fue de $2,41 \mathrm{~mm}$, mientras que en el caso del lado izquierdo fue de 4,24 mm (Fig. 3.B y C). Debido a la ubicación de los supernumerarios (inclinados, cercanos a dientes vecinos) y su proximidad con el foramen mental, se decide proceder con la exodoncia para luego realizar preservación del reborde alveolar e instalación de los implantes dentales.

\section{DISCUSIÓN}

Para la pesquisa e identificación de dientes supernumerarios, además del historial clínico, el examen radiográfico es esencial (Brook, 1975; Mali et al., 2012; Thumati et al., 2014), más aún para el caso de pacientes sindrómicos (Wang et al., 2007; Lubinsky \& Kantaputra), con antecedentes familiares de hiperdoncia (Skrinjaric \& Barac-Furtinovic, 1991; Umweni \& Osunbor, 2002; Wang et al.), historial de anomalías dentarias en dentición primaria (Skrinjaric \& Barac-Furtinovic; Whittington \& Durward, 1996; Mukhopadhyay \& Mitra, 2014) o que presenten simultáneamente alguna otra alteración dentaria. De acuerdo con Peker et al. (2009), en un 8,1\% de los casos de hiperodoncia se observó también microdoncia y dilaceración en molares. En nuestro caso estudio, al ser un paciente asintomático, sin historial ni manifestaciones clínicas evidentes de supernumerarios, la radiografía panorámica permitió el hallazgo de estos dientes que posteriormente fueron evaluados a través de CBCT. Distintos autores recomiendan este último tipo de examen para la planificación de tratamiento y determinación de posición y relaciones de los dientes supernumerarios con estructuras adyacentes vecinas. De acuerdo con Chou et al. (2015) es recomendable el uso rutinario de CBCT en pacientes con dientes supernumerarios.

La prevalencia de dientes supernumerarios en población no sindrómica es variable y depende del área geográfica, etnia y forma de muestreo de la población estudiada (Davis, 1987; Bäckman \& Wahlin, 2001; Altug-Atac \& Erdem, 2007; Kramer et al., 2008; Bereket et al., 2015; Chou et al.; Jammal Dobles \& Silva Meza, 2015; Basalamah \& Baroudi, 2016). Por 
otro lado, se han reportado diferentes porcentajes de prevalencia de dientes supernumerarios tanto para niños como para adultos. En este ámbito, Kramer et al. estudiaron una muestra de niños brasileños y encontraron una prevalencia de supernumerarios de $0,3 \%$, similar a lo reportado por Basalamah \& Baroudi quienes estudiaron niños de Yemen. Bereket et al. reporta una prevalencia de $0,79 \%$ en una población turca. Por su parte Bäckman \& Wahlin reportan una prevalencia de $1,9 \%$ en niños suecos, mientras que Skrinjaric $\&$ Barac-Furtinovic, reportan una prevalencia de $0,1 \%$ en niños de Croacia. La prevalencia parece similar y un tanto mayor en poblaciones de occidente, tal y como lo demuestran los estudios de Davis, en niños de Hong Kong, y Chou et al., en una población taiwanesa, quienes reportan una prevalencia de $2,7 \%$ y $2,6 \%$ respectivamente. En el caso de poblaciones americanas, Jammal Dobles \& Silva Meza reportan una prevalencia de 2,8 \% en una población mexicana, mientras que Simões et al. (2011) reporta una prevalencia de $1,7 \%$ en una población brasileña.

Gran parte de los estudios coincide en que el sitio más frecuente en donde se presentan los supernumerarios es el maxilar, con una predilección por el sexo masculino (Davis; Jammal Dobles \& Silva Meza; Bereket et al.; Anegundi et al., 2014), siendo el mesiodens el diente observado de mayor frecuencia (Bäckman \& Wahlin; Bereket $e t$ al.; Anegundi et al.), seguido por los cuartos molares y en tercer lugar los premolares (Bereket et al.). Yusof (1990) señala que presentar dientes supernumerarios múltiples en pacientes no asociados a síndromes es infrecuente y concluye que, en caso de presentarse, el sitio más recurrente es en la región de premolares mandibulares, tal como sucede con nuestro paciente al presentar de forma bilateral premolares supernumerarios.

Esta anomalía puede pasar desapercibida sin presentar sintomatología ni complicaciones, sin embargo, también puede tener implicancias clínicas adversas, las cuales deben ser abordadas rápidamente. Entre estas complicaciones tenemos las alteraciones en la erupción y posición de los dientes primarios o permanentes, inclusiones dentarias con o sin impactación, diastemas, malposiciones dentarias, reabsorciones radiculares, dilaceración y desarrollo de quistes foliculares (Garvey et al.; Ata-Ali et al., 2014). En este reporte, los dientes supernumerarios no erupcionaron y permanecieron incluidos, provocando malposición dentaria, adelgazamiento de tablas óseas, además de una leve rizálisis del diente 4.5. La planificación del tratamiento en estos casos, variará de acuerdo a cada paciente; algunos autores recomiendan realizar exodoncias de manera profiláctica para evitar complicaciones futuras, otros autores recomiendan esperar a que los dientes adyacentes terminen su desarrollo radicular para evitar injurias durante la cirugía. Garvey et al. entregan algunas recomendaciones sobre cuando es adecuado monitorear o realizar la exodoncia y para esto último indican que se debe intervenir si se ha retrasado la erupción de un diente permanente, si se ha producido desplazamiento de incisivos centrales, si existe patología asociada, por tratamiento de ortodoncia, si interfiere en el tratamiento con implantes o si ha erupcionado de forma espontánea (Garvey et al.; Thumati et al.). Omer et al. estiman que un tiempo conveniente para la exodoncia de los dientes supernumerarios maxilares no erupcionados es entre los 6 y 7 años, después de ese tiempo aumentaría la probabilidad de complicaciones. A pesar de estas propuestas, no existe consenso sobre cuál es el momento adecuado para intervenir quirúrgicamente. En este reporte, la planificación del tratamiento considera la necesidad de exodoncia de los dientes supernumerarios y la colocación de implantes como tratamiento rehabilitador para devolver tanto estética como funcionalidad.

\section{CONCLUSIÓN}

Los dientes supernumerarios son alteraciones del desarrollo en donde hay un aumento en el número normal de dientes, su prevalencia es mayor en hombres, en dentición permanente, en el maxilar, y son más frecuentes en un patrón unilateral. Pueden provocar diversas complicaciones en la dentición adyacente como rizálisis, rotación dentaria, diastemas, apiñamiento, quistes foliculares y dilaceración entre otros. Aún no está claro el momento exacto para intervenir quirúrgicamente, sin embargo, los autores coinciden que se debe actuar lo más rápido posible en caso de detectar daño a alguna estructura. En este reporte, la decisión clínica consistió en la exodoncia de los dientes supernumerarios, debido a su malposición e interferencia para el correcto posicionamiento de los implantes y al daño a estructuras vecinas que habían ocasionado. Un examen clínico exhaustivo con el apoyo de exámenes complementarios imagenológicos es imprescindible para el diagnóstico y planificación del tratamiento.

FUENTES, R.; ÁLVAREZ, G.; GARAY, I.; ARIAS, A. \& DIAS, F. J. Supplementary supernumerary teeth in a partially edentulous adult patient: Case report. Int. J. Morphol., 36(1):478-482, 2018.

SUMMARY: Cases in which the normal amount of teeth is exceeded for the primary or permanent dentition formula, are known as supernumerary teeth or hyperdontia. This can occur in single, or multiple form, unilaterally or bilaterally, and affect both maxilla and mandible. It is more prevalent in men, in permanent dentition and in the maxilla; the mesiodens type being the most common supernumerary. Its etiology is still not entirely clear, however, hyperactivity of the dental lamina associated to genetic 
and environmental factors is the most accepted theory. Supernumerary teeth can be asymptomatic and can only be detected in radiographs. They can also present various clinical manifestations such as dental malposition, delay in dental eruption, root resorption and diastemas. Treatment depends on the type of tooth and its relation with adjacent structures. In the following report we present the case of an asymptomatic, upper and lower partially edentulous 54-year-old male patient, who requested rehabilitation treatment at a private dental clinic in Temuco, Chile. In routine panoramic radiography, two supernumerary teeth were observed, one on each side of the mandible and in the premolar region. Upon reviewing these findings with cone beam computed tomography, it was determined that the supernumerary on the right side corresponded to a supplementary premolar and the supernumerary on the left side corresponded to a supernumerary of molariform morphology. In this case we determined to extract the teeth, due to their location and impact on subsequent rehabilitation treatment.

KEY WORDS: Supernumerary teeth; Hyperdontia; Premolar; Supplementary tooth.

\section{REFERENCIAS BIBLIOGRÁFICAS}

Altug-Atac, A. T. \& Erdem, D. Prevalence and distribution of dental anomalies in orthodontic patients. Am. J. Orthod. Dentofacial Orthop., 131(4):510-4, 2007

Anegundi, R. T.; Tegginmani, V. S.; Battepati, P.; Tavargeri, A.; Patil, S.; Trasad, V. \& Jain, G. Prevalence and characteristics of supernumerary teeth in a non-syndromic South Indian pediatric population. J. Indian Soc. Pedod. Prev. Dent., 32(1):9-12, 2014.

Ata-Ali, F.; Ata-Ali, J.; Peñarrocha-Oltra, D. \& Peñarrocha-Diago, M. Prevalence, etiology, diagnosis, treatment and complications of supernumerary teeth. J. Clin. Exp. Dent., 6(4):e414-8, 2014.

Bäckman, B. \& Wahlin, Y. B. Variations in number and morphology of permanent teeth in 7-year-old Swedish children. Int. J. Paediatr. Dent., 11(1):11-7, 2001

Basalamah, M. \& Baroudi, K. Prevalence of oro-dental anomalies among schoolchildren in Sana'a city, Yemen. East Mediterr. Health J., 22(1):338, 2016.

Bereket, C.; Çakır-Özkan, N.; S, ener, I ' .; Bulut, E. \& Bastan, A. I ' . Analyses of 1100 supernumerary teeth in a nonsyndromic Turkish population: A retrospective multicenter study. Niger. J. Clin. Pract., 18(6):731-8, 2015.

Brook, A. H. Variables and criteria in prevalence studies of dental anomalies of number, form and size. Community Dent. Oral Epidemiol., 3(6):28893, 1975.

Chou, S. T.; Chang, H. P.; Yang, Y. H.; Lung, C. Y.; Tseng, Y. C.; Pan, C. Y. \& Cheng, J. H. Characteristics of supernumerary teeth among nonsyndromic dental patients. J. Dent. Sci., 10(2):133-8, 2015.

Davis, P. J. Hypodontia and hyperdontia of permanent teeth in Hong Kong schoolchildren. Community Dent. Oral Epidemiol., 15(4):218-20, 1987.

Garvey, M. T.; Barry, H. J. \& Blake, M. Supernumerary teeth--an overview of classification, diagnosis and management. J. Can. Dent. Assoc., 65(11):612-6, 1999

Ge, L. H. \& Wang, X. Recent advances in molecular mechanisms of supernumerary tooth formation. Beijing Da Хие Хие Bao., 45(4):661$5,2013$.

Jammal Dobles, N. \& Silva Meza, R. Prevalencia de dientes supernumerarios en una muestra mexicana. Rev. Mex. Ortod., 3(2):88-91, 2015.

Juuri, E. \& Balic, A. The biology underlying abnormalities of tooth number in humans. J. Dent. Res., 96(11):1248-56, 2017.
Kramer, P. F.; Feldens, C. A.; Ferreira, S. H.; Spiguel, M. H. \& Feldens, E. G. Dental anomalies and associated factors in 2- to 5-year-old Brazilian children. Int. J. Paediatr. Dent., 18(6):434-40, 2008.

Lubinsky, M. \& Kantaputra, P. N. Syndromes with supernumerary teeth. Am. J. Med. Genet. A, 170(10):2611-6, 2016.

Mali, S.; Karjodkar, F. R.; Sontakke, S. \& Sansare, K. Supernumerary teeth in non-syndromic patients. Imaging Sci. Dent., 42(1):41-5, 2012.

Montasser, M. A. \& Taha, M. Prevalence and distribution of dental anomalies in orthodontic patients. Orthodontics (Chic.), 13(1):52-9, 2012.

Mukhopadhyay, S. \& Mitra, S. Anomalies in primary dentition: Their distribution and correlation with permanent dentition. J. Nat. Sci. Biol. Med., 5(1):139-43, 2014.

Omer, R. S.; Anthonappa, R. P. \& King, N. M. Determination of the optimum time for surgical removal of unerupted anterior supernumerary teeth. Pediatr. Dent., 32(1):14-20, 2010.

Peker, I.; Kaya, E. \& Darendeliler-Yaman, S. Clinic and radiographical evaluation of non-syndromic hypodontia and hyperdontia in permanent dentition. Med. Oral Patol. Oral Cir. Bucal, 14(8):e393-7, 2009.

Primosch, R. E. Anterior supernumerary teeth--assessment and surgical intervention in children. Pediatr. Dent., 3(2):204-15, 1981.

Sebastián Sebastián, C.; Izquierdo Hernández, B.; Gutiérrez Alonso, C. \& Aso Vizán, A. Dientes supernumerarios: claves esenciales para un adecuado informe radiológico. Rev. Argent. Radiol., 80(4):258-67, 2016.

Simões, F. X. P. C.; Crusoé-Rebello, I.; Neves, F. S.; Oliveira-Santos, C.; Ciamponi, A. L. \& da Silva Filho, O. G. Prevalence of supernumerary teeth in orthodontic patients from southwestern Brazil. Int. J. Odontostomat., 5(2):199-202, 2011.

Skrinjaric, I. \& Barac-Furtinovic, V. Anomalies of deciduous teeth and findings in permanent dentition. Acta Stomatol. Croat., 25(3):151-6, 1991.

Thumati, P.; David, C. M. \& Tiwari, R. Non-syndromic multiple supernumerary teeth: A case report and review of literature. IJSS Case Rep. Rev., 1(5):1-5, 2014.

Umweni, A. A. \& Osunbor, G. E. Non-syndrome multiple supernumerary teeth in Nigerians. Odontostomatol. Trop., 25(99):43-8, 2002.

Valmaseda-Castellón, E.; Berini-Aytés, L. \& Gay-Escoda, C. Supernumerary premolars. Report of 10 cases. Bull. Group Int. Rech. Sci. Stomatol. Odontol., 43(1):19-25, 2001.

Wang, X. X.; Zhang, J. \& Wei, F. C. Autosomal dominant inherence of multiple supernumerary teeth. Int. J. Oral Maxillofac. Surg., 36(8):756$8,2007$.

Whittington, B. R. \& Durward, C. S. Survey of anomalies in primary teeth and their correlation with the permanent dentition. N. Z. Dent. J., 92(407):4-8, 1996.

Yusof, W. Z. Non-syndrome multiple supernumerary teeth: literature review. J. Can. Dent. Assoc., 56(2):147-9, 1990.

Dirección para correspondencia

Dr. Ramón Fuentes Fernández

Centro de Investigación en Ciencias Odontológicas CICO

Facultad de Odontología

Universidad de La Frontera

Av. Francisco Salazar 01145

Temuco

CHILE

E-mail: ramon.fuentes@ufrontera.cl

Recibido : 07-12-2017

Aceptado: 31-01-2018 